\title{
Complete set of deformation potentials for AlN determined by reflectance spectroscopy under uniaxial stress
}

\section{$\operatorname{AUTHOR}(S)$ :}

Ishii, Ryota; Kaneta, Akio; Funato, Mitsuru;

Kawakami, Yoichi

\section{CITATION:}

Ishii, Ryota ...[et al]. Complete set of deformation potentials for AlN determined by reflectance spectroscopy under uniaxial stress. Physical Review B 2013, 87(23): 235201.

\section{ISSUE DATE:}

2013-06-04

URL:

http://hdl.handle.net/2433/175498

RIGHT:

(C) 2013 American Physical Society 


\title{
Complete set of deformation potentials for AIN determined by reflectance spectroscopy under uniaxial stress
}

\author{
Ryota Ishii, ${ }^{*}$ Akio Kaneta, Mitsuru Funato, and Yoichi Kawakami ${ }^{\dagger}$ \\ Department of Electronic Science and Engineering, Kyoto University, Kyoto 615-8510, Japan
}

(Received 23 March 2013; published 4 June 2013)

\begin{abstract}
Reflectance spectroscopy was performed for nonpolar and semipolar bulk aluminum nitride (AlN) substrates under uniaxial stress. The exciton-polariton theory was applied to interpret the reflectivity spectra, while the BirPikus deformation potential theory was used to analyze the stress dependence of exciton transition energies. This approach allowed all the deformation potentials in AlN to be determined without the quasicubic approximation.
\end{abstract}

DOI: 10.1103/PhysRevB.87.235201

PACS number(s): 71.70.Fk, 71.55.Eq, 71.35.-y

\section{INTRODUCTION}

Nitride semiconductors have received much attention as candidates for solid-state light sources in the deep-ultraviolet (DUV) spectral region due to a number of advantages, including robustness, harmlessness, and wavelength tunability. ${ }^{1}$ Aluminum nitride (AlN), which has an energy gap of $\sim 6.0 \mathrm{eV}$, is a key material to realize DUV optoelectronic devices.

Light emitting diodes (LEDs) have been demonstrated using AlN $p$ - $i$ - $n$ structure $^{2}$ or AlGaN/AlN heterostructures ${ }^{3,4}$ in the DUV spectral region. However, the external quantum efficiency of AlN-based DUV-LEDs remains low compared to GaN-based LEDs in the blue or blue-green spectral region. ${ }^{5}$ Moreover, the shortest wavelength of laser diodes (LDs) has been limited to $336 \mathrm{~nm}$ at room temperature. ${ }^{6}$ To realize highly efficient DUV-LEDs or even shorter-wavelength DUV-LDs, the electronic properties of AIN must be thoroughly understood and both the crystalline quality and device fabrication processes must be improved.

The valence band structure of AlN differs significantly from those of conventional wurtzite semiconductors, such as GaN. The topmost valence band in AlN has an irreducible representation of $\Gamma_{7}^{\mathrm{v}}$ due to the giant negative crystal-field splitting energy, ${ }^{7}$ whereas the lower valence bands are weakly split into $\Gamma_{9}^{\mathrm{V}}$ and $\Gamma_{7}^{\mathrm{V}}$ due to the spin-orbit interaction. On the other hand, from low to high electron energy, the valence band ordering in $\mathrm{GaN}$ is $\Gamma_{7}^{\mathrm{v}}-\Gamma_{7}^{\mathrm{v}}-\Gamma_{9}^{\mathrm{v}} \cdot{ }^{8}$ Consequently, when the mixed alloy of $\mathrm{Al}_{x} \mathrm{Ga}_{1-x} \mathrm{~N}$ is formed, the highest valence band may switch from $\Gamma_{9}^{\mathrm{v}}$ to $\Gamma_{7}^{\mathrm{v}}$ at a certain Al composition $x$. However, theoretically estimating the critical composition $x$ is difficult because the crystal-field splitting and the spin-orbit splitting energy in AlN remain controversial despite numerous theoretical $^{7,9-14}$ and experimental ${ }^{15-19}$ studies. Furthermore, it has been experimentally demonstrated that strain-induced effects and quantum-confinement effects significantly alter the critical composition $x$ in $c$-plane AlGaN films ${ }^{20,21}$ and $c$ plane AlGaN/AlN quantum wells. ${ }^{22-25}$ Both these effects have been theoretically investigated using an empirical calculation method. ${ }^{26-28}$ However, due to the ambiguity of the material parameters of AlN, it is difficult to reach a definite conclusion.

The Bir-Pikus theory, ${ }^{29,30}$ which uses deformation potentials $\left(C_{i=1-6}\right)$ as the essential material parameters, can well describe the strain-induced effects in electronic band structures. However, few studies have experimentally determined the deformation potentials in AlN. ${ }^{19,31}$ In addition, the few reported works only extract the deformation potentials of
$C_{1-4}$ under the assumption of the quasicubic approximation. ${ }^{29}$ The remaining deformation potentials, $C_{5}$ and $C_{6}$, are indispensable parameters to predict the electronic band structures of nonpolar ${ }^{32}$ and semipolar AlGaN/AlN heterostructures, in which the internal electric field should be small. ${ }^{33}$ Furthermore, our recent experimental work indicates that the quasicubic approximation breaks down between $C_{3}$ and $C_{4}$ in $\mathrm{GaN}$, and we predict a deviation from the quasicubic relation in AlN. ${ }^{34}$ Consequently, all the deformation potentials in AlN should be experimentally investigated without assuming that the quasicubic approximation holds.

In this paper, reflectance spectroscopy is performed for $m$-plane and $r$-plane AlN substrates under uniaxial stress. The excitonic resonances clearly depend on the uniaxial stress. The exciton-polariton theory is applied to interpret the reflectivity spectra, while the Bir-Pikus deformation potential theory is used to analyze the stress dependence of exciton transition energies. Herein we determine all the deformation potentials in AlN without the quasicubic approximation. In addition, we determine the crystal-field interaction, spin-orbit interaction, and electron-hole exchange interaction energy. These findings provide a deeper understanding and may realize more reliable modeling of AlN-based optoelectronic devices.

\section{EXPERIMENT}

\section{A. General}

The samples used in this study were $m$-plane and $r$-plane AlN substrates grown by the physical vapor transport method. The samples were cut into cuboid shapes along the crystal axes, and mechanically lapped until optically flat edge surfaces were obtained. Typical dimensions of the samples were $3 \times 3 \times 0.5 \mathrm{~mm}^{3}$. The sample was set in the same uniaxial stress-loading apparatus used in our previous work. ${ }^{34}$ The incident light was linearly polarized by a Glan-Thompson prism. The reflectivity spectra were calibrated by a reference sample on a (0001) sapphire substrate. The experiments were performed at a normal incidence condition at nearly $4.2 \mathrm{~K}$. Figure 1 summarizes the experimental configurations. The refractive index of air, $n_{\text {air }}=1.000310$, was used to transform wavelength into energy. In this paper, uniaxial stress and an electric polarization vector are defined as $P$ and $E$, respectively. 
(a)

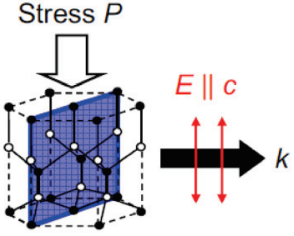

(b)

(c) Stress $P$

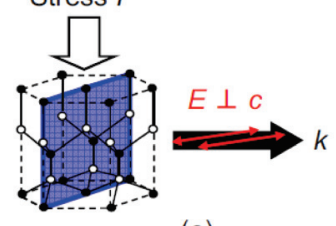

(d)
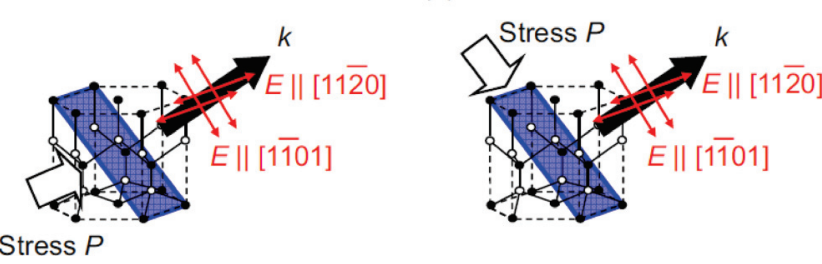

FIG. 1. (Color online) Experimental configurations (a) $P \| c$, $E \| c$, (b) $P \perp c, E \| c$, (c) $P \| c, E \perp c$, (d) $P \perp c, E \|[11 \overline{2} 0]$ or $E \|$ [1101], and (e) $P \|$ [11 101$], E \|[11 \overline{2} 0]$ or $E \|$ [1 101$]$, where $k$ is a Poynting vector.

\section{B. The A exciton}

For the A-exciton $\left(\Gamma_{7}^{c} \otimes \Gamma_{9}^{\mathrm{V}}=\Gamma_{1}^{\mathrm{A}} \oplus \Gamma_{2}^{\mathrm{A}} \oplus \Gamma_{5}^{\mathrm{A}}\right.$, which is the lowest energy transition) study, reflectance spectroscopy under uniaxial stress was performed using a $30 \mathrm{~W}$ deuterium lamp as the incident light. The reflection signals were dispersed by a $50 \mathrm{~cm}$ spectrometer with a holographic grating ( 2400 grooves $/ \mathrm{mm}$ ) and detected by a charge-coupled device cooled with liquid nitrogen. The experimental setup had a resolution of about $0.045 \mathrm{~nm}$.

Polarized reflectance measurements were performed on the $m$-plane bulk AIN substrate under uniaxial stress parallel to

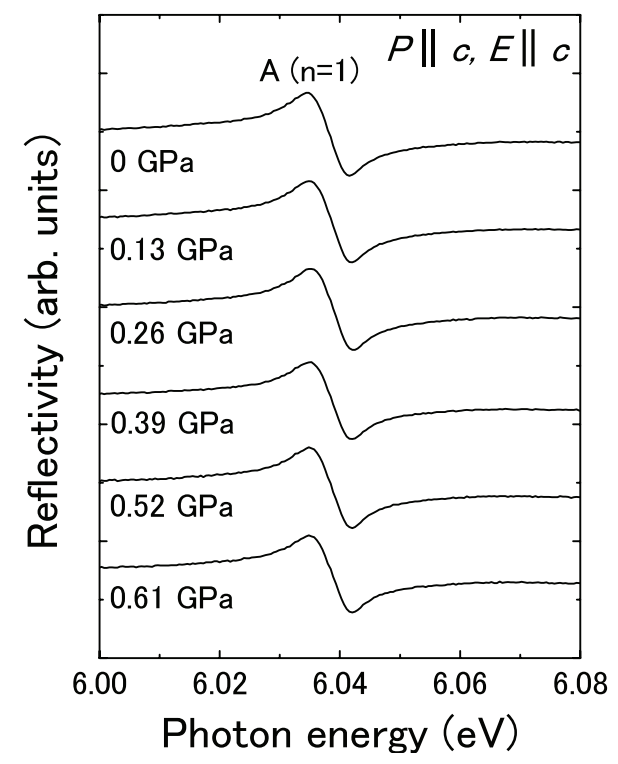

FIG. 2. Reflectance spectra of the $m$-plane bulk AlN substrate under uniaxial stress $P\|c, E\| c$.

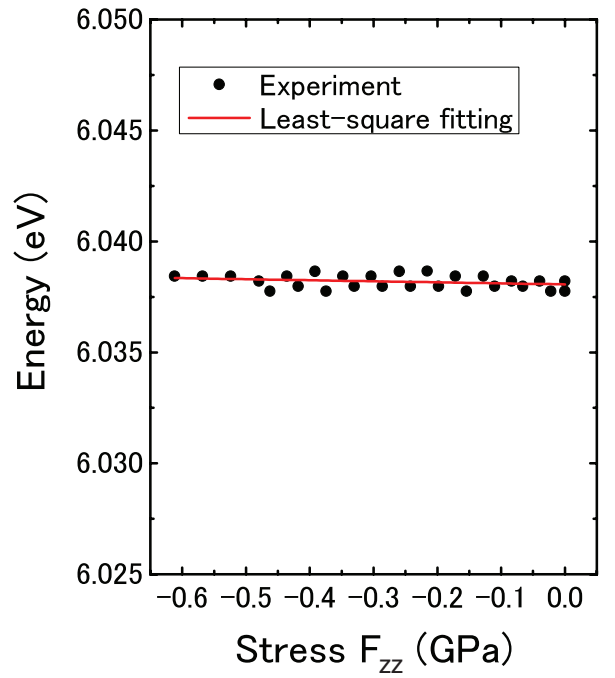

FIG. 3. (Color online) Uniaxial stress dependence of the Aexciton transition energy for $P\|c, E\| c$.

the $c$ axis and the electric polarization vector parallel to the $c$ axis ( $P\|c, E\| c)$. Figures 1(a) and 2 show the experimental configuration and result, respectively. As plotted in Fig. 3, the A-exciton energy in this configuration is almost independent of the uniaxial stress. If a reflection anomaly is composed of an exciton, the stress dependence of the exciton transition energy is well approximated by that of $E_{a v}$ and $E\left(R_{a v}\right)$, as defined in Fig. 4. Herein, $E^{\mathrm{A}}\left(R_{a v}\right)$ is substituted for the A-exciton transition energy due to the better definition. The least-square fit with a linear function yields

$$
\frac{\partial E^{\mathrm{A}}\left(R_{a v}\right)}{\partial F_{z z}}=-0.5 \mathrm{meV} / \mathrm{GPa},
$$

where $F_{z z}$ is the stress tensor along the $c$ axis. The fitted line is also depicted in Fig. 3.

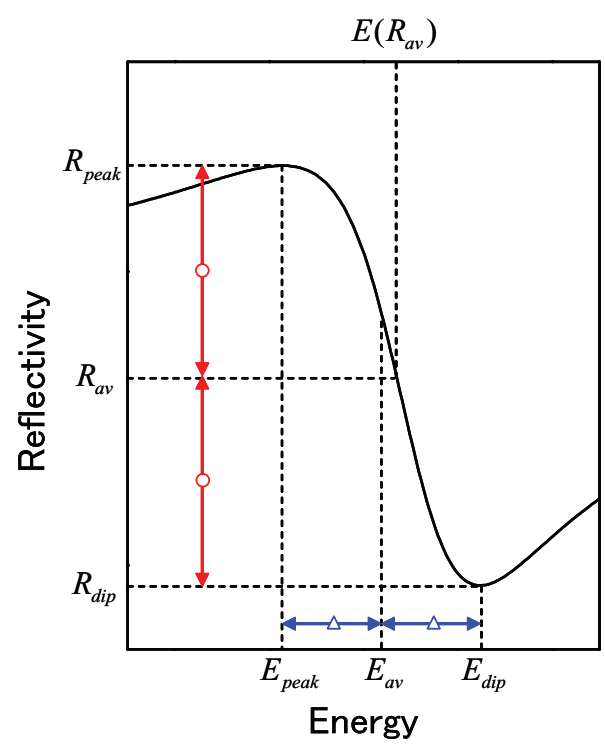

FIG. 4. (Color online) Definition of the characteristic energies in the reflection anomaly. 


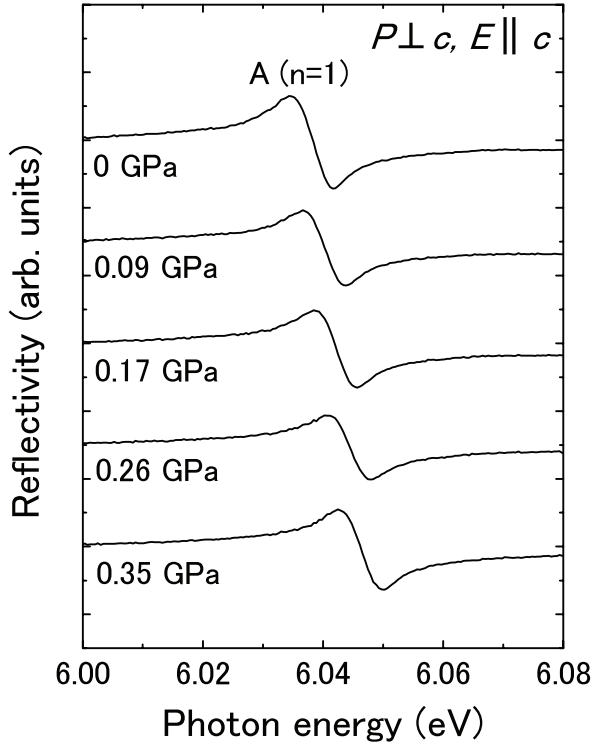

FIG. 5. Reflectance spectra of the $m$-plane bulk AlN substrate under uniaxial stress $P \perp c, E \| c$.

Polarized reflectance measurements were performed on the $m$-plane bulk AlN substrate under uniaxial stress perpendicular to the $c$ axis and the electric polarization vector parallel to the $c$ axis $(P \perp c, E \| c)$. Figures 1 (b) and 5 show the experimental configuration and result, respectively. Uniaxial stress causes the A-exciton transition energy to shift to higher energies in this configuration. Figure 6 plots both the experimental data and least-square fit of the uniaxial stress dependence of the A-exciton transition energy. In this case, the derivative of the energy by the stress is obtained as

$$
\frac{\partial E^{\mathrm{A}}\left(R_{a v}\right)}{\partial F_{x x}}=-21.8 \mathrm{meV} / \mathrm{GPa},
$$

where $F_{x x}$ is the stress tensor along the $a$ axis.

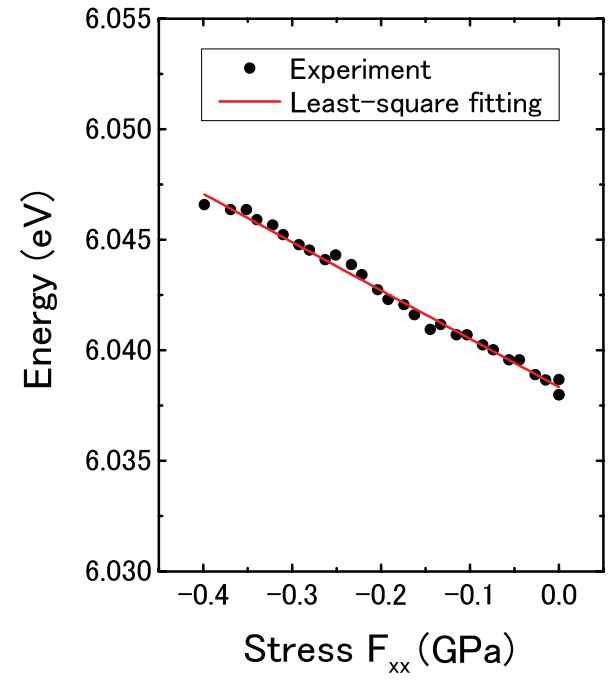

FIG. 6. (Color online) Uniaxial stress dependence of the Aexciton transition energy for $P \perp c, E \| c$.

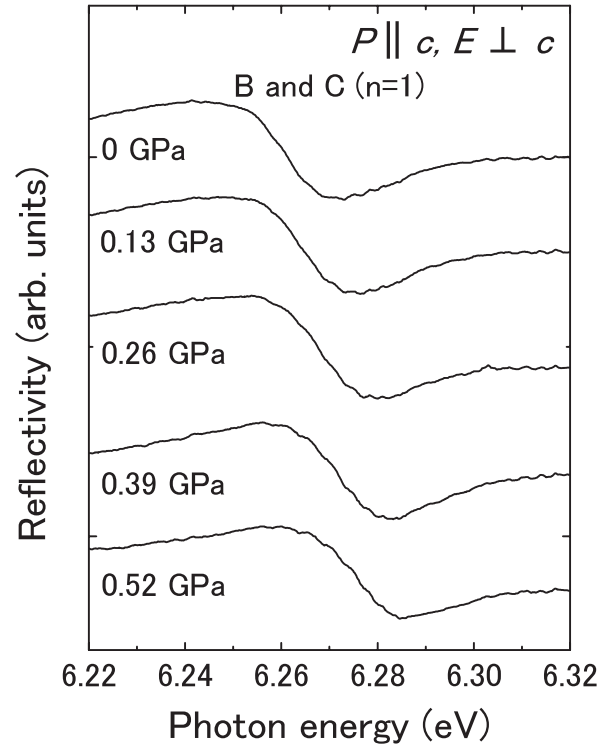

FIG. 7. Reflectance spectra of the $m$-plane bulk AlN substrate under uniaxial stress $P \| c, E \perp c$.

\section{The $B$ and $C$ excitons}

To enhance the light intensity in the DUV spectral region in the B-exciton $\left(\Gamma_{7}^{c} \otimes \Gamma_{9}^{\mathrm{v}}=\Gamma_{5}^{\mathrm{B}} \oplus \Gamma_{6}^{\mathrm{B}}\right)$ and $\mathrm{C}$-exciton $\left(\Gamma_{7}^{c} \otimes\right.$ $\Gamma_{7}^{\mathrm{v}}=\Gamma_{1}^{\mathrm{C}} \oplus \Gamma_{2}^{\mathrm{C}} \oplus \Gamma_{5}^{\mathrm{C}}$ ) studies, a laser driven light source (EQ99, ENERGETIQ) served as the incident light. The optical path was fully purged by dry nitrogen. The reflection signals were dispersed by a $1-\mathrm{m}$ spectrometer with a blazed-holographic grating $\left(2400\right.$ grooves $\left./ \mathrm{mm}, \lambda_{\mathrm{BL}}=250 \mathrm{~nm}\right)$ and detected by a charge-coupled device cooled with liquid nitrogen. The experimental setup had a resolution of about $0.012 \mathrm{~nm}$.

Polarized reflectance measurements were performed on the $m$-plane bulk AlN substrate under uniaxial stress parallel to the $c$ axis and the electric polarization vector perpendicular to the $c$ axis $(P \| c, E \perp c)$. Figures 1 (c) and 7 show the experimental configuration and result, respectively. Because

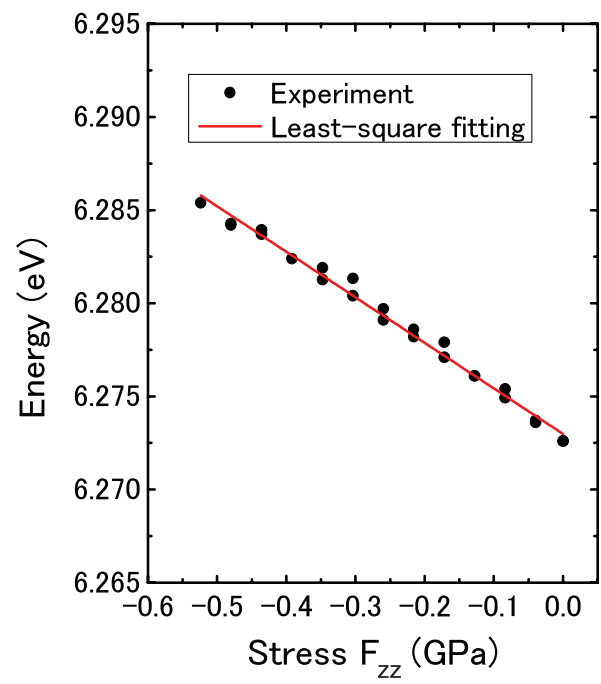

FIG. 8. (Color online) Uniaxial stress dependence of the reflection minimum composed of $\mathrm{B}$ and $\mathrm{C}$ excitons for $P \| c, E \perp c$. 


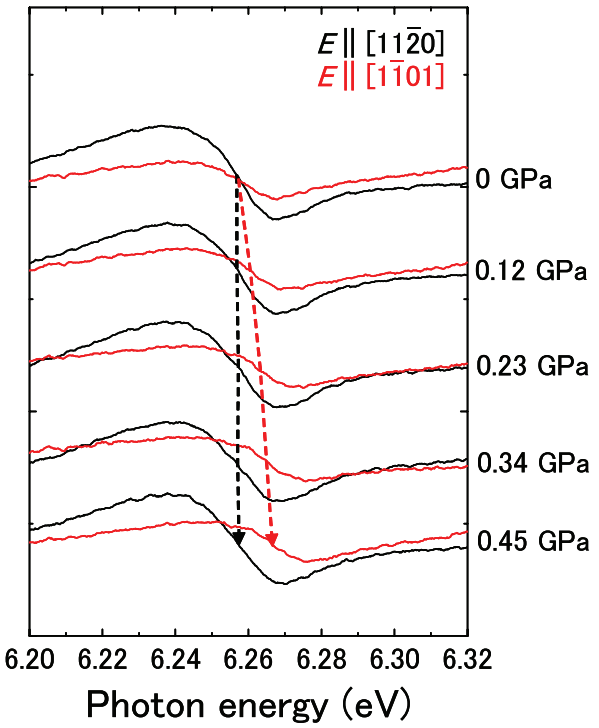

FIG. 9. (Color online) Reflectance spectra of the $r$-plane bulk AlN substrate under uniaxial stress $P \| c$. Results for both electric polarizations are summarized.

it is difficult to extract the precise value of $R_{\text {peak }}$, the reflection minimum was used as the characteristic energy in this case. The uniaxial stress has a negligible influence on the energy difference between the B- and C-exciton transition energies, and equally shifts their energies. The physics are discussed later. Consequently, the stress dependence of the $\mathrm{B}$ and $\mathrm{C}$ excitons are assumed to be equivalent. Figure 8 plots the uniaxial stress dependence of $E_{\mathrm{dip}}^{\mathrm{B}, \mathrm{C}}$ from the spectra and the least-square fit. The derivative of the energy by the stress is obtained as

$$
\frac{\partial E_{\mathrm{dip}}^{\mathrm{B}, \mathrm{C}}}{\partial F_{z z}}=-24.5 \mathrm{meV} / \mathrm{GPa} .
$$

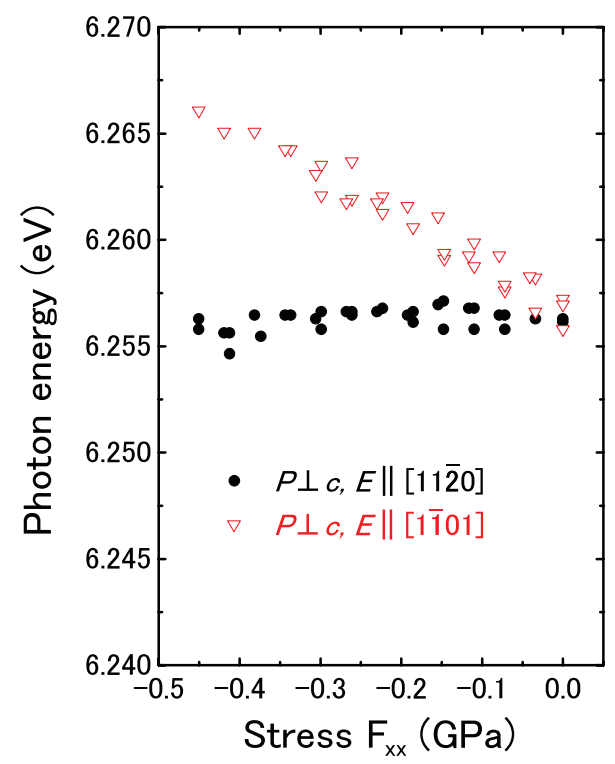

FIG. 10. (Color online) Uniaxial stress dependence of $E\left(R_{\mathrm{av}}\right)$ composed of $\mathrm{B}$ and $\mathrm{C}$ excitons for $P \perp c$.

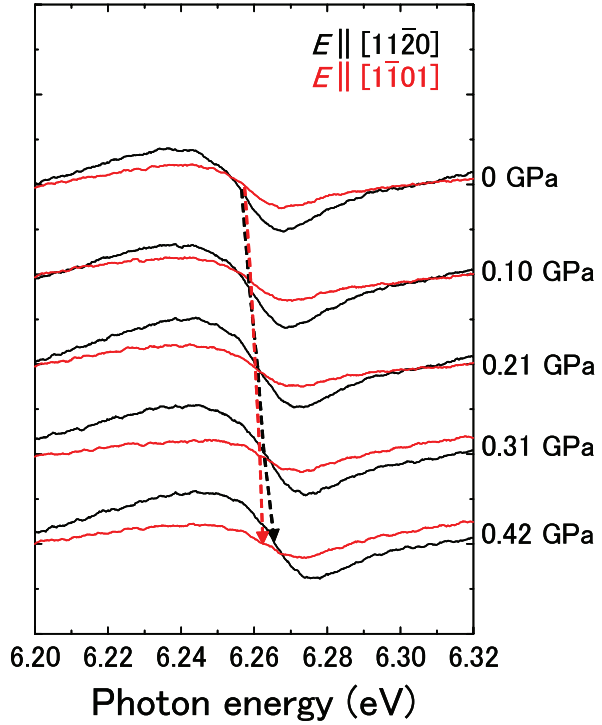

FIG. 11. (Color online) Reflectance spectra of the $r$-plane bulk AlN substrate under uniaxial stress $P \|[1 \overline{1} 01]$. Results for both electric polarizations are summarized.

Next, polarized reflectance measurements were performed for $r$-plane bulk AlN substrates. The stress direction was perpendicular to the $c$ axis $(P \perp c)$. Figure 1(d) shows the experimental configuration. Figure 9 shows the experimental results of both electric polarizations $(E \|[11 \overline{2} 0]$ and $E \|$ [1101]). $E \|[11 \overline{2} 0]$ and $E \|[1 \overline{1} 01]$ polarizations exhibit different stress dependences, suggesting that the reflection anomaly must be composed of more than one eigenstate. We attribute the eigenstates to $\Gamma_{5}^{\mathrm{B}}$ and $\Gamma_{5}^{\mathrm{C}}$ due to the small oscillator strength of $\Gamma_{6}^{\mathrm{B}}, \Gamma_{1}^{\mathrm{C}}$, and $\Gamma_{2}^{\mathrm{C}}$. Applying anisotropic stress reveals that $\mathrm{B}$ and $\mathrm{C}$ excitons exist in the reflection anomaly. It should be noted that their existence has not been directly demonstrated via experiments but only suggested by indirect procedures such as fitting analysis. Figure 10 plots the stress dependence of the resonance, where the characteristic energy is defined by $E\left(R_{a v}\right)$.

Finally, polarized reflectance measurements were performed for $r$-plane bulk AlN substrates where the stress was applied parallel to the [11 101$]$ direction. Figure 1(e) shows the experimental configuration. Figure 11 shows the experimental results of both electric polarizations $(E \|[11 \overline{2} 0]$ and $E \|$ [1101]). Shear stress was applied in this configuration. ${ }^{34}$ Figure 12 plots the stress dependence of the resonance using $E\left(R_{a v}\right)$.

\section{ANALYSIS}

\section{A. General}

Here, the methods to determine the deformation potentials in AlN are explained. The effective Hamiltonian for $1 s$ excitons in Wurtzite crystals is expressed as ${ }^{29}$

$$
\begin{aligned}
H_{\mathrm{ex}}= & \Delta_{1} J_{z}^{2}+\Delta_{2} J_{z} \sigma_{v z}+\Delta_{3}\left(\sigma_{+v} J_{-}+\sigma_{-v} J_{+}\right) \\
& +\left(C_{1}+C_{3} J_{z}^{2}\right) \epsilon_{z z}+\left(C_{2}+C_{4} J_{z}^{2}\right)\left(\epsilon_{x x}+\epsilon_{y y}\right) \\
& -C_{5}\left(J_{-}^{2} \epsilon_{+}+J_{+}^{2} \epsilon_{-}\right)-2 C_{6}\left(\left[J_{z} J_{+}\right] \epsilon_{-z}+\left[J_{z} J_{-}\right] \epsilon_{+z}\right) \\
& +E_{\text {bind }}+\frac{1}{2} j\left(\sigma_{e} \cdot \sigma_{h}\right) .
\end{aligned}
$$




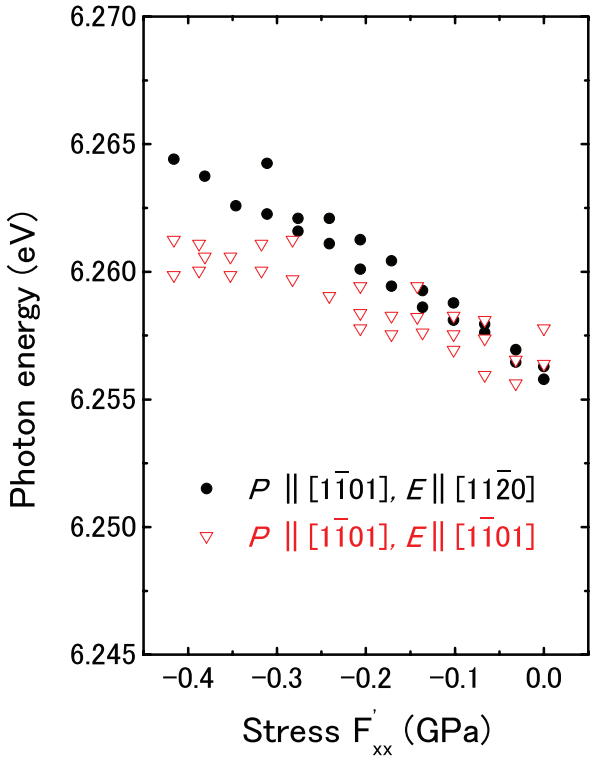

FIG. 12. (Color online) Uniaxial stress dependence of $E\left(R_{a v}\right)$ composed of $\mathrm{B}$ and $\mathrm{C}$ excitons for $P \|[1 \overline{1} 01]$.

$E_{\text {bind }}$ is an exciton binding energy and the other notations in Eq. (4) are the same as those in Ref. 34. In this paper, $C_{1,2}$ and $C_{3,4}$ are called the hydrostatic and uniaxial deformation potentials, respectively. Likewise, $C_{5}$ and $C_{6}$ are called the anisotropic and shear deformation potentials, respectively. The hydrostatic deformation potentials in AlN were initially determined, and then the remaining parameters were extracted.

\section{B. Hydrostatic deformation potentials}

Previous studies have experimentally determined the hydrostatic and uniaxial deformation potentials in AlN as follows. ${ }^{19,31}$ A series of $c$-plane AlN thin films with biaxial strain was prepared by varying the film thickness, and optical spectroscopic measurements were performed to experimentally elucidate the relation between the exciton transition energies and the strain. The relationship is theoretically expressed by only two parameters,

$$
\begin{aligned}
& \delta^{h}=C_{1}-\frac{C_{33}}{C_{13}} C_{2}, \\
& \delta^{u}=C_{3}-\frac{C_{33}}{C_{13}} C_{4},
\end{aligned}
$$

where $C_{i j}$ denote the elastic stiffness constants. $\delta^{h}$ and $\delta^{u}$ are determined by comparing experiments and theory. Obviously, the deformation potentials cannot be determined by only these equations, even if the elastic stiffness constants are known. Therefore, part of the quasicubic approximation ${ }^{29}$ is usually assumed:

$$
\begin{gathered}
C_{1}=C_{2}-C_{3}, \\
C_{3}=-2 C_{4} .
\end{gathered}
$$

Combining Eqs. (5)-(8) allows the hydrostatic and uniaxial deformation potentials in AlN to be experimentally determined. ${ }^{19,31}$

This paper adopts a different approach, in which uniaxial stress conditions are utilized. Herein, the excitonic structures

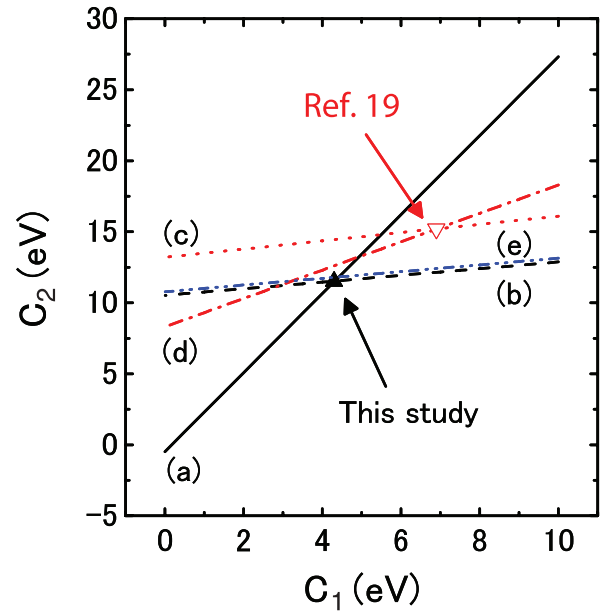

FIG. 13. (Color online) Relation between $C_{1}$ and $C_{2}$ (a) Black solid line: $P \| c$ in our experiment; (b) black dashed line: $P \perp c$ in our experiment; (c) red dotted line: biaxial strain conditions in Ref. 19; (d) red single-chain line: the quasicubic approximation in Ref. 19; (e) blue double-chain line: reanalysis of the experimental results in Ref. 19.

of AlN must be recalled. The A-exciton transition energy vastly differs from the $\mathrm{B}$ - and $\mathrm{C}$-exciton transition energies (see Figs. 2 and 9). Therefore, the mixing terms between them are so small that the angular momentum of the A exciton can be regarded as a good quantum number $\left(J_{z}=0\right)$. Applying this assumption to Eq. (4) allows the strain-related terms of the effective Hamiltonian for the A exciton to be expressed as

$$
H_{\mathrm{ex}}^{\mathrm{A}}=C_{1} \epsilon_{z z}+C_{2}\left(\epsilon_{x x}+\epsilon_{y y}\right) .
$$

Subsequently, the derivative of the A-exciton energy by the uniaxial stress $(P \| c, P \perp c)$ can be written as

$$
\begin{gathered}
-\frac{\partial E^{\mathrm{A}}}{\partial F_{z z}}=S_{33} C_{1}+2 S_{13} C_{2}, \\
-\frac{\partial E^{\mathrm{A}}}{\partial F_{x x}}=S_{13} C_{1}+\left(S_{11}+S_{12}\right) C_{2},
\end{gathered}
$$

where $S_{i j}$ indicate the elastic compliance constants. Therefore, if the elastic constants are known, the hydrostatic deformation potentials in AlN can be determined by only the uniaxial stress dependence of the A exciton along the two perpendicular directions. It should be noted that this approach does not require the quasicubic approximation.

Figure 13 shows the relation between $C_{1}$ and $C_{2}$ satisfying Eqs. (10) and (11) using the elastic constants determined by Brillouin spectroscopy on bulk AlN substrates. ${ }^{35}$ From our experiments, the hydrostatic deformation potentials in AlN are $C_{1}=4.3 \mathrm{eV}$ and $C_{2}=11.5 \mathrm{eV}$, which is the intersection of lines (a) and (b) in Fig. 13. Additionally, lines (c) and (d) are from Ref. 19, and the values of $C_{1}$ and $C_{2}$ were determined by the intersection of (c) and (d); the hydrostatic deformation potentials in AIN were extracted using the elastic constants determined by a first-principles study. ${ }^{36}$ Although the elastic constants have been frequently used in theoretical calculations of AlN-based heterostructures, if the experimental results in Ref. 19 are reanalyzed using the elastic constants in Ref. 35, 
TABLE I. Exciton deformation potentials (eV) in AlN.

\begin{tabular}{|c|c|c|c|c|c|c|c|}
\hline AlN & $C_{1}$ & $C_{2}$ & $C_{3}$ & $C_{4}$ & $C_{5}$ & $C_{6}$ & $C_{3} /\left(-2 C_{4}\right)$ \\
\hline \multicolumn{8}{|l|}{ Experimental: } \\
\hline Ikeda et al..$^{\mathrm{a}}$ & 8.4 & 15.6 & 8.19 & -4.10 & & & 1 \\
\hline $\mathrm{Gil}^{\mathrm{b}}$ & 6.04 & -2.15 & 8.19 & -4.10 & & & 1 \\
\hline Rossbach et al. ${ }^{\mathrm{c}}$ & 6.9 & 15.2 & 8.3 & -4.15 & & & 1 \\
\hline Present work & 4.3 & 11.5 & 6.8 & -3.6 & -2.8 & -4.5 & 0.9 \\
\hline \multicolumn{8}{|l|}{ Calculation: } \\
\hline Suzuki et al. ${ }^{\mathrm{d}}$ & & & 4.49 & -2.18 & -2.58 & -4.08 & 1.0 \\
\hline Kim et al..$^{\mathrm{e}}$ & & & 9.6 & -4.8 & & & 1.0 \\
\hline Shimada et al. ${ }^{\mathrm{f}}$ & & & 8.84 & -3.92 & -3.36 & & 1.1 \\
\hline Wagner et al. ${ }^{\mathrm{g}}$ & 3.39 & 11.81 & 9.42 & -4.02 & & & 1.2 \\
\hline Vurgaftman et al. ${ }^{\mathrm{h}}$ & 3.4 & 11.8 & 8.8 & -3.9 & -3.4 & -3.4 & 1.1 \\
\hline Vurgaftman et al..$^{\mathrm{i}}$ & 3.4 & 11.8 & 9.4 & -4.0 & -3.3 & -2.7 & 1.2 \\
\hline Yan et al. ${ }^{\mathrm{j}}$ & 4.21 & 12.07 & 9.22 & -3.74 & -3.30 & -4.49 & 1.2 \\
\hline
\end{tabular}

a Reference 31.

${ }^{\mathrm{b}}$ Reference 37.

${ }^{\mathrm{c}}$ Reference 19.

${ }^{\mathrm{d}}$ Reference 38 .

${ }^{\mathrm{e}}$ Reference 10

${ }^{\mathrm{f}}$ Reference 11.

${ }^{\mathrm{g}}$ Reference 13 .

${ }^{\mathrm{h}}$ Reference 39.

${ }^{\mathrm{i}}$ Reference 40.

${ }^{\mathrm{j}}$ Reference 14 .

line (e) is obtained in Fig. 13. Surprisingly, line (e) sweeps the hydrostatic deformation potentials of $C_{1}$ and $C_{2}$ that we determined. The results indicate that both the experimental results under uniaxial stress (this work) and under biaxial strain (Ref. 19) can be consistently described by our hydrostatic deformation potentials and the elastic constants in Ref. 35. It should be noted that if the elastic constants in Ref. 36 are used, there is not a solution of $C_{1}$ and $C_{2}$ which can explain both the results. Table I summarizes the hydrostatic deformation potentials in AlN.

\section{Bulk parameters}

$\Delta_{1}, \Delta_{2}, \Delta_{3}$, and $j$ in Eq. (4) are called bulk parameters, and are determined in this section. Section II C demonstrates the existence of the $\mathrm{B}$ and $\mathrm{C}$ excitons in the reflection anomaly observed in Figs. 9 and 11. The reflectivity spectra must be carefully resolved into the components of $\Gamma_{5}^{\mathrm{B}}$ and $\Gamma_{5}^{\mathrm{C}}$ excitonic states to precisely determine the bulk parameters. Herein, we analyze the reflectivity spectra using the dielectric function of exciton polaritons as

$$
\epsilon(E)=\epsilon_{b}+\sum_{i} \frac{\chi_{i} E_{i}}{E_{i}^{2}-E^{2}-i E \Theta_{i}},
$$

where $\epsilon_{b}$ is a background dielectric constant. $E_{i}, \Theta_{i}$, and $\chi_{i}$ are the eigenenergy, the empirical broadening width, and the oscillator strength of $i$ th transitions, respectively. The dielectric function for $E \|[1 \overline{1} 01]$ is expressed as

$$
\epsilon_{[1 \overline{1} 01]}=\epsilon_{[0001]} \sin ^{2} \theta+\epsilon_{[11 \overline{2} 0]} \cos ^{2} \theta,
$$

where $\theta$ is the tilt angle from the $c$ plane $^{34}\left(\theta=42.8^{\circ}\right.$ in this case). For $\epsilon_{[0001]}(E \|[0001])$, the A-exciton transition with the 2LO-phonon and the A-exciton continuum are considered, ${ }^{19}$ where the stress dependence of the longitudinal optic (LO)-phonon energy is neglected. ${ }^{41}$ For $\epsilon_{[11 \overline{2} 0]}(E \|[11 \overline{2} 0])$, the B- and C-exciton transitions are considered.

It should be noted that the spectral fitting without guidelines does not directly yield a unique solution, which is why the bulk parameters of AIN have yet to be precisely determined. The energy difference between $E\left(R_{a v}\right)$ and $E_{a v}$ should be noted; the $E\left(R_{a v}\right)$ is $\sim 4 \mathrm{meV}$ higher than the $E_{a v}$ for the reflection anomaly in the $E \perp c$ configurations (Figs. 7, 9 , and 11), in which the B- and C-exciton transitions are involved. Obviously, the energy difference is larger than the experimental resolution. Based on the analysis of Eq. (12), to describe such an energy difference between $E\left(R_{a v}\right)$ and $E_{a v}$, the oscillator strength of $\Gamma_{5}^{\mathrm{C}}$ must be several times larger than that of $\Gamma_{5}^{\mathrm{B}}$, and is due to the positive electron-hole exchange interaction energy $j$ in Eq. (4). Our photoluminescence study of $c$-plane homoepitaxial AlN films ${ }^{42}$ supports this interpretation, although a negative electron-hole exchange interaction energy has been reported in the literature. ${ }^{43}$ Herein, the spectral fitting yields bulk parameters of $\Delta_{1}=-211.5 \mathrm{meV}, \Delta_{2}=6.0 \mathrm{meV}$, $\Delta_{3}=6.5 \mathrm{meV}$, and $j=6.0 \mathrm{meV}$. Table II summarizes the bulk parameters in AlN.

\section{Uniaxial, anisotropic, and shear deformation potentials}

$$
\text { 1. } P \| c, E \perp c
$$

The uniaxial stress dependence in Fig. 8 was analyzed. Section III B describes how the angular momentum of the A exciton can be regarded as $J_{z}=0$. Similarly, the angular momentum of the $\mathrm{B}$ and $\mathrm{C}$ excitons can be well approximated 
TABLE II. Bulk parameters in AlN in units of meV.

\begin{tabular}{|c|c|c|c|c|}
\hline AlN (Experimental) & $\Delta_{1}$ & $\Delta_{2}$ & $\Delta_{3}$ & $j$ \\
\hline Chen et al. ${ }^{\mathrm{a}}$ & -230 & 6.7 & 6.7 & \\
\hline Silveira et al..$^{\mathrm{b}}$ & -225 & 12 & 12 & \\
\hline Prinz et al..$^{\mathrm{c}}$ & -237 & 6.7 & 6.7 & \\
\hline Ikeda et $a l .{ }^{\mathrm{d}}$ & -152.4 & 6.3 & 6.3 & \\
\hline Taniyasu et al. ${ }^{\mathrm{e}}$ & -165 & 6.3 & 6.3 & \\
\hline Rossbach et al. ${ }^{\mathrm{f}}$ & -212 & 5.3 & 5.3 & \\
\hline Feneberg et al..$^{\mathrm{g}}$ & & & & -4.0 \\
\hline Present work & -211.5 & 6.0 & 6.5 & 6.0 \\
\hline
\end{tabular}

${ }^{\mathrm{a}}$ Reference 16.

${ }^{\mathrm{b}}$ Reference 17.

${ }^{\mathrm{c}}$ Reference 44.

${ }^{\mathrm{d}}$ Reference 31 .

${ }^{\mathrm{e}}$ Reference 45

${ }^{\mathrm{f}}$ Reference 19.

${ }^{\mathrm{g}}$ Reference 43 .

by $J_{z}= \pm 1$. Under this assumption, an interaction does not cause mixing between the $\mathrm{B}$ and $\mathrm{C}$ excitons for $P \| c$. Subsequently, the strain-related terms of the B- and C-exciton Hamiltonian can be written as

$$
H_{\mathrm{ex}}^{\mathrm{B}, \mathrm{C}}=\left(C_{1}+C_{3}\right) \epsilon_{z z}+\left(C_{2}+C_{4}\right)\left(\epsilon_{x x}+\epsilon_{y y}\right) .
$$

The uniaxial stress dependences of the $\mathrm{B}$ and $\mathrm{C}$ excitons for $P \| c$ are equivalent, and the derivative of the energy by the uniaxial stress is expressed as

$$
-\frac{\partial E^{\mathrm{B}, \mathrm{C}}}{\partial F_{z z}}=S_{33}\left(C_{1}+C_{3}\right)+2 S_{13}\left(C_{2}+C_{4}\right) .
$$

Combining Eqs. (3) and (15), the first relation between uniaxial deformation potentials is obtained as

$$
\alpha^{u}=C_{3} S_{33}+2 C_{4} S_{13}=24.0 \mathrm{meV} / \mathrm{GPa},
$$

where $\alpha^{u}$ is the same as in Ref. 34 .

\section{2. $P \perp c, E \|[11 \overline{2} 0]$ or $E \|[1 \overline{101}]$}

The uniaxial stress dependence of $E\left(R_{a v}\right)$ in Fig. 10 was analyzed. When the uniaxial stress is applied perpendicular to the $c$ axis, the stress dependence of the $\mathrm{B}$ exciton differs from that of the $\mathrm{C}$ exciton due to stress-induced mixing. Therefore, the simple analysis used until this point cannot be used here. An alternative approach must be adopted.

In the adopted approach, the exciton effective Hamiltonian was numerically solved assuming a certain set of $C_{3-5}$, where the bulk parameters, hydrostatic deformation potentials, and elastic constants were fixed. Then the eigenenergies and the oscillator strength of all the excitons were obtained. Substituting these values for Eq. (12) and assuming certain values for the remaining parameters theoretically produced the reflectivity spectra. Subsequently, we calculated the value of $E\left(R_{a v}\right)$ for the reflection anomaly composed of the $\mathrm{B}$ and $\mathrm{C}$ excitons. These processes were repeated until $C_{3-5}$ minimized the error between experiments and theory.

Before the fitting procedures, the fitting parameters such as $\epsilon_{b}$ or $\chi_{i}$ in Eq. (12) were examined. To reproduce the experimental reflectivity spectra by Eq. (12), the appropriate values must be substituted into these parameters. The examination shows that these values are almost independent of $C_{3-5}$. Therefore, in the fitting procedures for $E \|$ [1120], we can reduce the number of fitting parameters to three, $C_{3-5}$. For $E \|[1 \overline{1} 01]$, the contributions from the $E \perp$ [0001] component were added. This significantly reduces the computational complexity and decreases the fitting ambiguity.

The fitting procedures were performed on the stress dependence in Fig. 10, yielding

$$
\begin{gathered}
\beta^{u}=C_{3} S_{13}+C_{4}\left(S_{11}+S_{12}\right)=-9.3 \mathrm{meV} / \mathrm{GPa}, \\
\beta^{a}=-C_{5}\left(S_{11}-S_{12}\right)=11.5 \mathrm{meV} / \mathrm{GPa},
\end{gathered}
$$

where $\beta^{u}$ and $\beta^{a}$ are the same as in Ref. 34. The second relation between $C_{3}$ and $C_{4}$ is obtained. Although $C_{5}$ can be deduced from Eq. (18), it is determined below.

\section{3. $P$ || [1101], $E \|[11 \overline{2} 0]$ or $E$ || [11̄01]}

The uniaxial stress dependence of $E\left(R_{a v}\right)$ in Fig. 12 was analyzed. The deformation potentials of $C_{3-6}$ were derived via the same method discussed in the previous section. The fitting procedures were performed on the stress dependences in Fig. 12, yielding

$$
\begin{aligned}
\gamma^{u}= & C_{3}\left(S_{13} \cos ^{2} \theta+S_{33} \sin ^{2} \theta\right) \\
& +C_{4}\left[\left(S_{11}+S_{12}\right) \cos ^{2} \theta+2 S_{13} \sin ^{2} \theta\right] \\
= & 3.6 \mathrm{meV} / \mathrm{GPa}, \\
\gamma^{a}=- & C_{5}\left(S_{11}-S_{12}\right) \cos ^{2} \theta=5.8 \mathrm{meV} / \mathrm{GPa}, \\
\gamma^{s}= & \frac{1}{2} C_{6} S_{44} \sin \theta \cos \theta=-12.5 \mathrm{meV} / \mathrm{GPa},
\end{aligned}
$$

where $\gamma^{u}, \gamma^{a}$, and $\gamma^{s}$ are the same as in Ref. 34. The third relation between $C_{3}$ and $C_{4}$ is obtained. $C_{5}$ and $C_{6}$ are determined below, but can also be deduced from Eqs. (20) and (21), respectively.

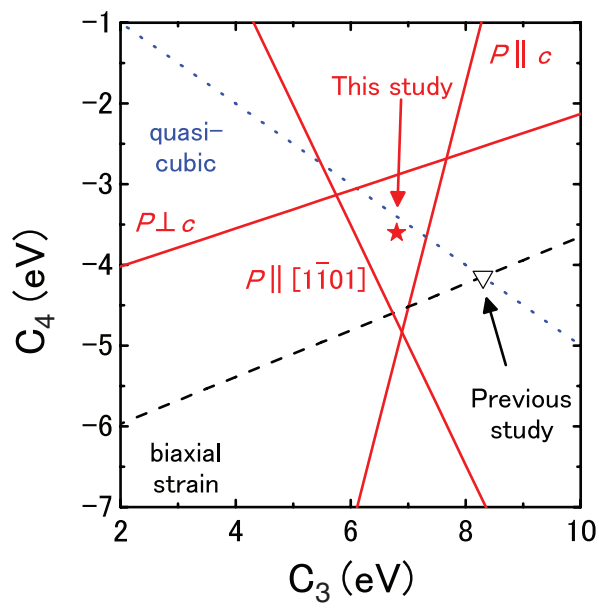

FIG. 14. (Color online) Relations between $C_{3}$ and $C_{4}$, obtained in our experiments under three different conditions: $P \| c, P \perp c$, and $P \|[1 \overline{1} 01]$. Values of $C_{3}$ and $C_{4}$ in Ref. 19 are also plotted. 
(a)

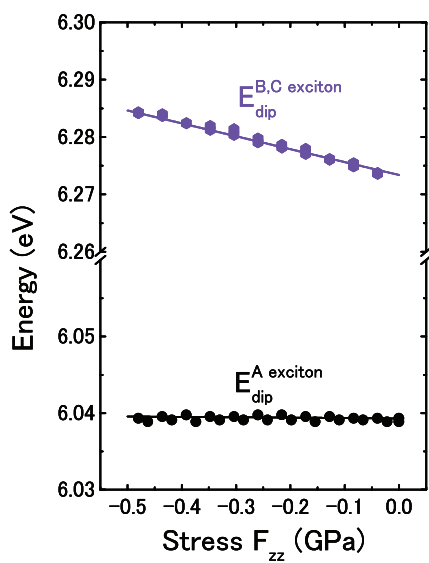

(b)

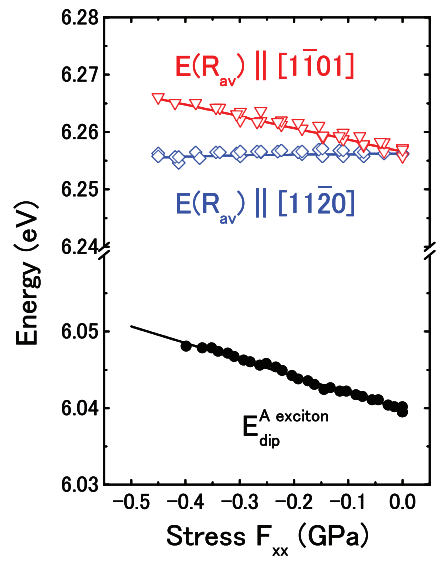

(c)

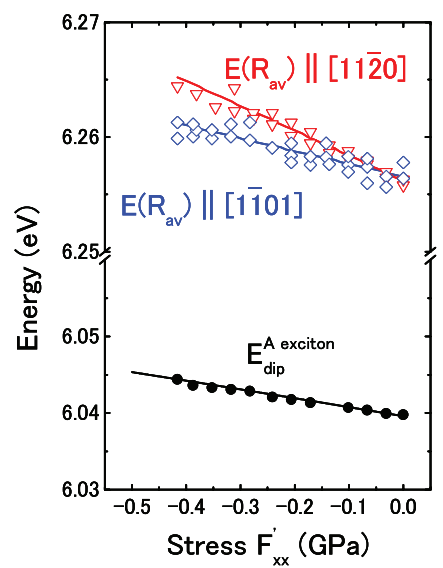

FIG. 15. (Color online) Comparison between the experimental (points) and theoretical (lines) stress dependence in each configuration (a) $P \| c$, (b) $P \perp c$, (c) $P \|[1 \overline{1} 01]$. Stress dependence of $E_{\mathrm{dip}}^{\mathrm{A}}$ is the same as that $E^{\mathrm{A}}\left(R_{a v}\right)$.

\section{Determination of uniaxial, anisotropic, and shear deformation potentials}

Thus far, we have obtained three equations for the relationship between uniaxial deformation potentials [Eqs. (16), (17), and (19)], two equations for anisotropic deformation potential [Eqs. (18) and (20)], and one equation for shear deformation potential [Eq. (21)]. To extract the four most plausible parameters $C_{3-6}$ from these six equations, $C_{3}$ and $C_{4}$ were initially determined because they were observed in all the experiments. Figure 14 plots the three relations between $C_{3}$ and $C_{4}$. These three relations form a triangle, and selecting the position close to the center simultaneously minimized the errors in all the experiments. Thus, the values determined for $C_{3}$ and $C_{4}$ are 6.8 and $-3.6 \mathrm{eV}$, respectively.

$\beta^{a}, \gamma^{a}$, and $\gamma^{s}$ were reevaluated using the least-squares fitting, where $\beta^{u}$ and $\gamma^{u}$ were fixed using the above values of $C_{3}$ and $C_{4}$. Subsequently, $C_{5}=-2.8 \mathrm{eV}$ and $C_{6}=-4.5 \mathrm{eV}$ are obtained.

Using the determined exciton deformation potentials, all the stress dependences were calculated. Figure 15 shows the results. All the experimental results are fully described by the deformation potentials that we determined. The errors of $C_{1,2}$ and $C_{3-6}$ are about $10 \%$ and $15 \%$, respectively. Table I summarizes all the deformation potentials in AlN.

\section{E. Quasicubic approximation in wurtzite crystals}

Figure 14 shows that the uniaxial deformation potentials in AlN do not rigorously satisfy the quasicubic approximation. In this final section, we consider the deviation from the quasicubic approximation in wurtzite crystals.

Here, we relate the deviation from the quasicubic approximation to the $c / a$ in wurtzite crystals. $c / a$ and $u$ can be mentioned as structural parameters in wurtzite crystals, where $a$ and $c$ are lattice constants and $u$ is an internal parameter. Generally, $c / a$ is inversely correlated with $u$. Herein, $c / a$ is chosen as the structural parameter. The deviation from the quasicubic approximation can be evaluated by the following equations:

$$
\xi=-\frac{C_{1}-C_{2}}{C_{3}}
$$

$$
\begin{gathered}
\zeta=-\frac{C_{3}}{2 C_{4}}, \\
\eta=\frac{C_{3}+4 C_{5}}{\sqrt{2} C_{6}} .
\end{gathered}
$$

If $\xi=\zeta=\eta=1$ holds, then the quasicubic approximation is satisfied. Table III summarizes the values of $c / a, u, \xi$, $\zeta$, and $\eta$ in wurtzite crystals, and shows that the quasicubic

TABLE III. $c / a, u, \xi, \zeta$, and $\eta$ in wurtzite crystals.

\begin{tabular}{lccccc}
\hline \hline Materials & $c / a$ & $u$ & $\xi$ & $\zeta$ & $\eta$ \\
\hline Experimental: & & & & & \\
$\mathrm{AlN}^{\mathrm{a}}$ & 1.6009 & & 1.1 & 0.9 & 0.7 \\
$\mathrm{ZnO}^{\mathrm{b}}$ & 1.6024 & & 0 & 0.3 & $|2.8|^{\mathrm{m}}$ \\
$\mathrm{ZnO}^{\mathrm{c}}$ & 1.6024 & & 0.2 & 0.5 & 3.8 \\
$\mathrm{CdS}^{\mathrm{d}}$ & 1.6235 & & 1.3 & 0.2 & $|2.8|^{\mathrm{m}}$ \\
$\mathrm{GaN}^{\mathrm{e}}$ & 1.6259 & & 1.0 & 0.5 & 1.4 \\
$\mathrm{CdSe}^{\mathrm{f}}$ & 1.6305 & & 0.7 & 0.9 & $|4.2|^{\mathrm{m}}$ \\
$\mathrm{Ideal}$ & 1.6330 & 0.3750 & & & \\
$\mathrm{Calculation:}^{\mathrm{Mgg}}$ & & & & & \\
$\mathrm{CdO}^{\mathrm{h}}$ & 1.5442 & 0.3919 & 1.0 & 1.5 & 0.4 \\
$\mathrm{AlN}^{\mathrm{i}}$ & 1.5715 & 0.3878 & 1.4 & -3.1 & 3.2 \\
$\mathrm{ZnO}^{\mathrm{j}}$ & 1.6025 & 03819 & 0.9 & 1.2 & 0.6 \\
$\mathrm{InN}^{\mathrm{k}}$ & 1.6047 & 0.3807 & -1.3 & 0.3 & 1.8 \\
$\mathrm{GaN}^{\mathrm{l}}$ & 1.6209 & 0.3796 & 0.4 & 0.8 & 1.3 \\
\hline \hline & 1.6257 & 0.3772 & 0.6 & 0.9 & 1.1 \\
\hline $\mathrm{Pren}^{\mathrm{m}}$ & & & & &
\end{tabular}

${ }^{\mathrm{a} P r e s e n t}$ work.

${ }^{\mathrm{b}}$ Reference 46.

${ }^{\mathrm{c}}$ Reference 47.

${ }^{\mathrm{d}}$ Reference 46.

${ }^{\mathrm{e}}$ Reference 34.

${ }^{\mathrm{f}}$ Reference 46.

${ }^{g}$ Reference 48.

${ }^{\mathrm{h}}$ Reference 48.

${ }^{\mathrm{i}}$ Reference 14.

${ }^{\mathrm{j}}$ Reference 48.

${ }^{\mathrm{k}}$ Reference 14.

${ }^{1}$ Reference 14.

${ }^{\mathrm{m}}$ The sign could not be determined. 
approximation in AlN breaks, especially for $\eta$. However, we cannot find an explicit relation between the deviation and $c / a$. To reach a definite conclusion, both experimental and theoretical studies are necessary. Thus, our conclusions from this study are the quasicubic approximation does not hold for AlN and it is not always applicable to wurtzite crystals.

\section{CONCLUSION}

In conclusion, reflectance spectroscopy under uniaxial stress reveals all the deformation potentials in AIN without the quasicubic approximation. In addition, the crystal-field interaction, the spin-orbit interaction, and the electron-hole exchange interaction are elucidated. These parameters can also explain previous experimental results. Furthermore, the experimental results indicate that a part of the quasicubic approximation is invalid for AlN.

\section{ACKNOWLEDGMENTS}

The authors would like to thank Jun Suda of Kyoto University. This research was partially supported by a Grantin-Aid for the Japan Society for the Promotion of Science as well as the Mizuho Foundation for the Promotion of Sciences. *ryota.ishii@optomater.kuee.kyoto-u.ac.jp

†kawakami@kuee.kyoto-u.ac.jp

${ }^{1}$ H. Morkoç, Handbook of Nitride Semiconductors and Devices (Wiley, Weinheim, 2008).

${ }^{2}$ Y. Taniyasu, M. Kasu, and T. Makimoto, Nature (London) 441, 325 (2006).

${ }^{3}$ H. Hirayama, S. Fujikawa, N. Noguchi, J. Norimatsu, T. Takano, K. Tsubaki, and N. Kamata, Phys. Status Solidi A 206, 1176 (2009). ${ }^{4}$ M. Shatalov, W. Sun, A. Lunev, X. Hu, A. Dobrinsky, Y. Bilenko, J. Yang, M. Shur, R. Gaska, C. Moe, G. Garrett, and M. Wraback, Appl. Phys. Express 5, 082101 (2012).

${ }^{5}$ Y. Narukawa, M. Ichikawa, D. Sanga, M. Sano, and T. Mukai, J. Phys. D: Appl. Phys. 43, 354002 (2010).

${ }^{6}$ H. Yoshida, Y. Yamashita, M. Kuwabara, and H. Kan, Appl. Phys. Lett. 93, 241106 (2008).

${ }^{7}$ M. Suzuki, T. Uenoyama, and A. Yanase, Phys. Rev. B 52, 8132 (1995).

${ }^{8}$ R. Dingle, D. D. Sell, S. E. Stokowski, and M. Ilegems, Phys. Rev. B 4, 1211 (1971).

${ }^{9}$ S. Wei and A. Zunger, Appl. Phys. Lett. 69, 2719 (1996).

${ }^{10}$ K. Kim, W. R. L. Lambrecht, B. Segall, and M. van Schilfgaarde, Phys. Rev. B 56, 7363 (1997).

${ }^{11}$ K. Shimada, T. Sota, and K. Suzuki, J. Appl. Phys. 84, 4951 (1998).

${ }^{12}$ S. K. Pugh, D. J. Dugdale, S. Brand, and R. A. Abram, Semicond. Sci. Technol. 14, 23 (1999).

${ }^{13}$ J. M. Wagner and F. Bechstedt, Phys. Rev. B 66, 115202 (2002).

${ }^{14}$ Q. Yan, P. Rinke, M. Winkelnkemper, A. Qteish, D. Bimberg, M. Scheffler, and C. G. Van de Walle, Semicond. Sci. Technol. 26, 014037 (2011).

${ }^{15}$ T. Onuma, S. F. Chichibu, T. Sota, K. Asai, S. Sumiya, T. Shibata, and M. Tanaka, Appl. Phys. Lett. 81, 652 (2002).

${ }^{16}$ J. Chen, W. Z. Shen, H. Ogawa, and Q. X. Guo, Appl. Phys. Lett. 85, 4334 (2004).

${ }^{17}$ E. Silveira, J. A. Freitas, Jr. O. J. Glembocki, G. A. Slack, and L. J. Schowalter, Phys. Rev. B 71, 041201(R) (2005).

${ }^{18}$ T. Onuma, T. Shibata, K. Kosaka, K. Asai, S. Sumiya, M. Tanaka, T. Sota, A. Uedono, and S. F. Chichibu, J. Appl. Phys. 105, 023529 (2009).

${ }^{19}$ G. Rossbach, M. Feneberg, M. Roppischer, C. Werner, N. Esser, C. Cobet, T. Meisch, K. Thonke, A. Dadgar, J. Blasing, A. Krost, and R. Goldhahn, Phys. Rev. B 83, 195202 (2011).

${ }^{20}$ K. B. Nam, J. Li, M. L. Nakarmi, J. Y. Lin, and H. X. Jiang, Appl. Phys. Lett. 84, 5264 (2004).
${ }^{21}$ H. Murotani, Y. Yamada, H. Miyake, and K. Hiramatsu, Appl. Phys. Lett. 98, 021910 (2011).

${ }^{22}$ H. Kawanishi, M. Senuma, and T. Nukui, Appl. Phys. Lett. 89, 041126 (2006).

${ }^{23}$ R. G. Banal, M. Funato, and Y. Kawakami, Phys. Rev. B 79, 121308(R) (2009).

${ }^{24}$ H. Hirayama, N. Noguchi, and N. Kamata, Appl. Phys. Express 3, 032102 (2010).

${ }^{25}$ T. M. Al Tahtamouni, J. Y. Lin, and H. X. Jiang, Appl. Phys. Lett. 101, 042103 (2012).

${ }^{26}$ A. A. Yamaguchi, Phys. Status Solidi C 5, 2364 (2008).

${ }^{27}$ T. K. Sharma, D. Naveh, and E. Towe, Phys. Rev. B 84, 035305 (2011).

${ }^{28}$ J. E. Northup, C. L. Chua, Z. Yang, T. Wunderer, M. Kneissl, N. M. Johnson, and T. Kolbe, Appl. Phys. Lett. 100, 021101 (2012).

${ }^{29}$ G. L. Bir and G. E. Pikus, Symmetry and Strain-Induced Effects in Semiconductors (Wiley, New York, 1974).

${ }^{30}$ W. H. Kleiner and L. M. Roth, Phys. Rev. Lett. 2, 334 (1959).

${ }^{31}$ H. Ikeda, T. Okamura, K. Matsukawa, T. Sota, M. Sugawara, T. Hoshi, P. Cantu, R. Sharma, J. F. Kaeding, S. Keller, U. K. Mishra, K. Kosaka, K. Asai, S. Sumiya, T. Shibata, M. Tanaka, J. S. Speck, S. P. DenBaars, S. Nakamura, T. Koyama, T. Onuma, and S. F. Chichibu, J. Appl. Phys. 102, 123707 (2007).

${ }^{32}$ M. Funato, K. Matsuda, R. G. Banal, R. Ishii, and Y. Kawakami, Phys. Rev. B 87, 041306(R) (2013).

${ }^{33} \mathrm{P}$. Waltereit, O. Brandt, A. Trampert, H. T. Grahn, J. Menniger, M. Ramsteiner, M. Reiche, and K. H. Ploog, Nature (London) 406, 865 (2000).

${ }^{34}$ R. Ishii, A. Kaneta, M. Funato, Y. Kawakami, and A. A. Yamaguchi, Phys. Rev. B 81, 155202 (2010).

${ }^{35}$ M. Kazan, E. Moussaed, R. Nader, and P. Masri, Phys. Status Solidi C 4, 204 (2007).

${ }^{36}$ A. F. Wright, J. Appl. Phys. 82, 2833 (1997).

${ }^{37}$ B. Gil, Phys. Rev. B 81, 205201 (2010).

${ }^{38}$ M. Suzuki and T. Uenoyama, J. Appl. Phys. 80, 6868 (1996).

${ }^{39}$ I. Vurgaftman and J. R. Meyer, J. Appl. Phys. 94, 3675 (2003).

${ }^{40}$ J. Piprek, Nitride Semiconductor Devices (Wiley-VCH, Weinheim, 2007), Chap. 2

${ }^{41}$ J. Gleize, M. A. Renucci, J. Frandon, E. Bellet-Amalric, and B. Daudin, J. Appl. Phys. 93, 2065 (2003).

${ }^{42}$ R. Ishii, M. Funato, and Y. Kawakami, Phys. Rev. B 87, 161204(R) (2013). 
${ }^{43}$ M. Feneberg, M. Romero, B. Neuschl, K. Thonke, M. Röppischer, C. Cobet, N. Esser, M. Bickermann, and R. Goldhahn, Appl. Phys. Lett. 102, 052112 (2013).

${ }^{44}$ G. M. Prinz, A. Ladenburger, M. Schirra, M. Feneberg, K. Thonke, R. Sauer, Y. Taniyasu, M. Kasu, and T. Makimoto, J. Appl. Phys. 101, 023511 (2007).

${ }^{45}$ Y. Taniyasu and M. Kasu, Appl. Phys. Lett. 98, 131910 (2011).
${ }^{46}$ D. W. Langer, R. N. Euwema, K. Era, and T. Koda, Phys. Rev. B 2, 4005 (1970).

${ }^{47}$ J. Wrzesinski and D. Fröhlich, Phys. Rev. B 56, 13087 (1997).

${ }^{48}$ Q. Yan, P. Rinke, M. Winkelnkemper, A. Qteish, D. Bimberg, M. Scheffler, and C. G. Van de Walle, Appl. Phys. Lett. 101, 152105 (2012). 